\section{International Scientific Journal Theoretical \& Applied Science}

\author{
p-ISSN: 2308-4944 (print) e-ISSN: 2409-0085 (online) \\ Year: $2016 \quad$ Issue: $10 \quad$ Volume: 42
}

Published: $30.10 .2016 \quad$ http://T-Science.org

SECTION 2. Applied mathematics. Mathematical modeling.
Denis Chemezov

Master of Engineering and Technology, Corresponding Member of International Academy of Theoretical and Applied Sciences, Lecturer of Vladimir Industrial College, Russian Federation chemezov-da@yandex.ru

Anzhelika Bayakina Lecturer of Vladimir Industrial College, Russian Federation bajakina.anzhelika@yandex.ru

\title{
THE SIMULATION OF THE FLUID FLOW ON THE INNER WALL OF THE CIRCULAR PIPE
}

Abstract: In the article is presented the analysis of computer simulation near-wall fluid flow in a circular pipe. Key words: wall, velocity, fluid, pipe.

Language: Russian

Citation: Chemezov D, Bayakina A (2016) THE SIMULATION OF THE FLUID FLOW ON THE INNER WALL OF THE CIRCULAR PIPE. ISJ Theoretical \& Applied Science, 10 (42): 1-3.

Soi: http://s-o-i.org/1.1/TAS-10-42-1 Doi: crossef http://dx.doi.org/10.15863/TAS.2016.10.42.1

\section{МОДЕЛИРОВАНИЕ ТЕЧЕНИЯ ЖИДКОСТИ НА ВНУТРЕННЕЙ СТЕНКЕ КРУГЛОЙ ТРУБЫ}

Аннотация: В статье представлен анализ компьютерного моделирования пристеночного течения жидкости в круглой трубе.

Ключевые слова: стенка, скорость, жидкость, труба.

\section{Introduction}

На этапе подготовки компьютерного моделирования процесса турбулентного потока задаются начальная скорость и направление течения жидкости и граничные условия для внутренней стенки модели трубы. Граничными условиями будут являться качество разбиения твердотельной объемной модели трубы на конечные элементы, величина скорости течения жидкости $v$ в пристеночном слое, величина шероховатости внутренней поверхности стенки трубы $B$ (закон стенки), характер скольжения жидкости на стенке трубы и др. параметры. Математическое описание изменения скорости турбулентного течения [1], а так же напряжения и деформации слоев жидкости в круглой трубе представлено в работе [2]. Для внутренней стенки модели трубы были заданы условия выполнения расчета турбулентного течения жидкости $[3$, с. 30-52] (рис. 1).
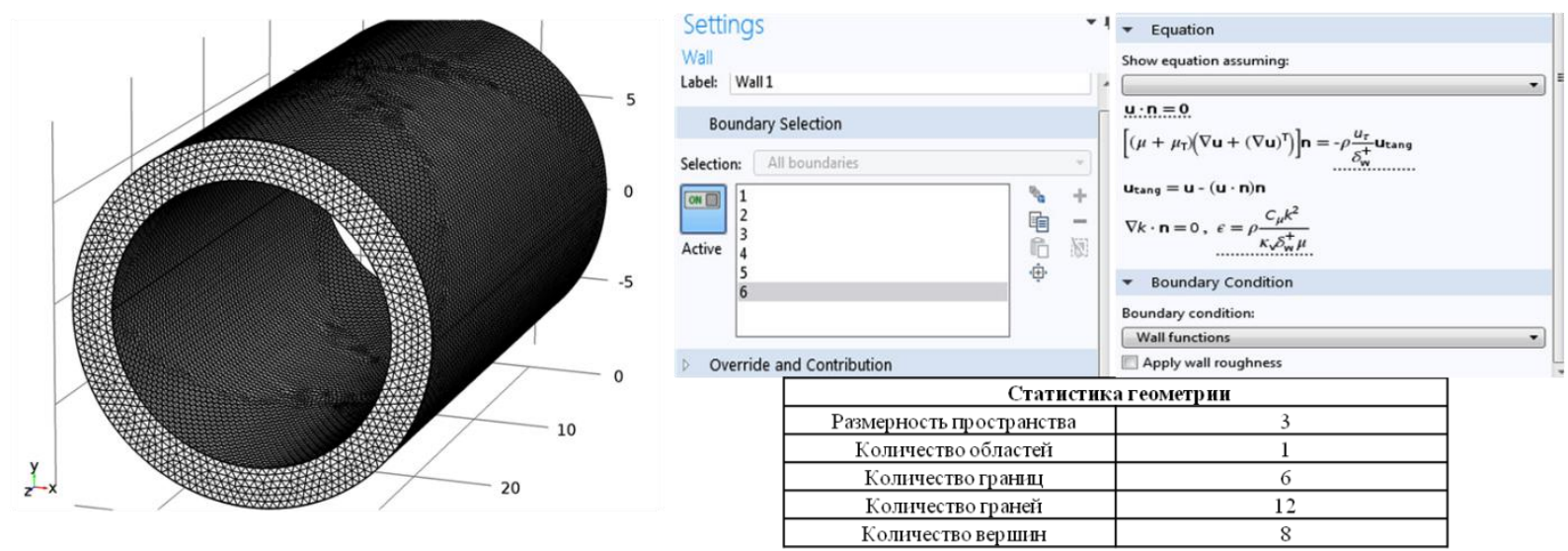

Рисунок 1 - Условия для внутренней стенки круглой трубы (модуль CFD программы COMSOL Multiphysics). 


\section{Materials and Methods}

Зависимость касательной компоненты скорости турбулентного течения жидкости от безразмерного расстояния до внутренней стенки трубы по нормали задается универсальным профилем (функцией). Выбор значений параметров для функции зависит от пристеночной функции. Пристеночная функция зависимость переменных в турбулентном пограничном слое от координаты по нормали к поверхности внутренней стенки круглой трубы. Пограничным слоем в данном случае будет являться некоторая область течения жидкости у поверхности стенки трубы, где наблюдается изменения скорости потока. В пограничном слое происходит плавное изменение скорости течения жидкости от максимального значения на верхней границе до нуля на нижней границе (стенка трубы).

Запишем формулу (1), определяющую промежуточную переменную для скорости течения жидкости на внутренней стенке модели круглой трубы $u_{n}, \mathrm{M} / \mathrm{c}$

$$
u_{n}=u \cdot n_{x}+v \cdot n_{y}+w \cdot n_{z},
$$

где $u, v, w$ - проекции вектора скорости потока соответственно на координатные оси $x, y, z ; n-$ нормальный вектор, $x, y$ и $z$ составляющие $n$.

Переменная $n_{i}-$ нормаль к некоторой площади поверхности конечных элементов внутренней стенки объемной модели круглой трубы. Так как скорость турбулентного потока жидкости в трубе изменяется со временем и не равна 0 , то для определения проекций вектора можно применить функции $u(x, y, z, t), v(x, y, z, t)$ и $w(x, y, z, t)$.

Составляющие $u_{n}(\mathrm{M} / \mathrm{c})$ можно представить в виде (2)

$$
\left\{\begin{array}{l}
u_{n x}=u_{n} \cdot n_{x} \\
u_{n y}=u_{n} \cdot n_{y} \\
u_{n z}=u_{n} \cdot n_{z}
\end{array}\right.
$$

где $u_{n x}, u_{n y}, u_{n z}-$ составляющие $u_{n}, \mathrm{~m} / \mathrm{c}$.

Скачок в нормальной скорости течения жидкости на внутренней стенке трубы будет равен промежуточной переменной, т. е. $u_{\text {jump }}=u_{n}, \mathrm{M} / \mathrm{c}$.

Составляющие тангенциальной скорости течения жидкости на внутренней стенке круглой трубы $u_{\text {tang. }}(\mathrm{M} / \mathrm{c})$ определяются по формулам (3)

$$
\left\{\begin{array}{l}
u_{\tan g . x}=u-n_{x}\left(u \cdot n_{x}+v \cdot n_{y}+w \cdot n_{z}\right) \\
u_{\tan g . y}=v-n_{y}\left(u \cdot n_{x}+v \cdot n_{y}+w \cdot n_{z}\right), \\
u_{\tan g . z}=w-n_{z}\left(u \cdot n_{x}+v \cdot n_{y}+w \cdot n_{z}\right)
\end{array}\right.
$$

где $u_{\text {tang. } x}, u_{\text {tang.y }}, u_{\text {tang. } z}-$ составляющие $u_{\text {tang. }}, \mathrm{M} / \mathrm{c}$.

По формуле (4) находят тангенциальную скорость течения жидкости на внутренней стенке круглой трубы $u^{+}$в единицах вязкости

$$
u^{+}=\frac{\ln \left(\delta_{w}^{+}\right)}{\kappa_{v}+B},
$$

где $\delta_{w}^{+}$- отрыв потока жидкости от внутренней стенки трубы в единицах вязкости, $\delta_{w}^{+}=11.06 ; \kappa_{v}$ - постоянный коэффициент Кармана, $\kappa_{v}=0.41$ [4]; $B$ - коэффициент, зависящий от величины шероховатости внутренней поверхности трубы [5].

Отрыв потока $\delta_{w}$ (м) происходит в результате прекращения движения жидкости вдоль поверхности внутренней стенки трубы (отсутствие вязкого трения и увеличение давления в направлении тока жидкости). $\delta_{w}$ находится из выражения (5)

$$
\delta_{w}=\frac{\delta_{w}^{+} \cdot[\mu]}{[\rho] \cdot \max \left(u_{\tau}, \sqrt{e p s}\right)},
$$

где $[\mu]$ и $[\rho]-$ оператор down для динамической вязкости (Па · с) и плотности жидкости $\left(к г / \mathrm{M}^{3}\right)$ соответственно, выбирает данные параметры на нижней границе компьютерного расчета [6, с. 7374]; max - математическая функция, возвращает большее из двух сравниваемых аргументов; $u_{\tau}$ скорость трения жидкости на внутренней стенке круглой трубы, м/с; eps - погрешность для операций над числами с плавающей точкой [7].

Функция $\max$ зачастую используется для ограничения нижней границы значений переменной. В условиях применения математической функции max следует избегать использования других функций в качестве параметров.

При моделировании турбулентного режима взаимодействие слоев жидкости с конечными элементами внутренней стенки круглой трубы происходит по касательной. Это взаимодействие является внешним трением жидкости на стенке трубы. Сила внешнего трения пропорциональна разности касательных скоростей слоев жидкости и площади конечных элементов внутренней стенки трубы. $u_{\tau}$ определяется по формуле (6)

$$
u_{\tau}=\max \left(C_{\mu}^{0.25} \cdot \sqrt{k_{\text {reg. }}}, \frac{\sqrt{u_{\tan g . x}^{2}+u_{\tan g . y}^{2}+u_{\tan g . z}^{2}+e p s}}{u^{+}}\right),
$$

где $C_{\mu}$ - параметр $k$ - $\varepsilon$ модели турбулентности (константа), $C_{\mu}=0.09$ [8]; $k_{\text {reg. }}$ - упорядоченная кинетическая энергия турбулентности потока 


\begin{tabular}{|c|c|c|c|c|c|c|}
\hline Impact Factor: & $\begin{array}{l}\text { ISRA (India) } \\
\text { ISI (Dubai, UAE } \\
\text { GIF (Australia) } \\
\text { JIF }\end{array}$ & $\begin{array}{l}=1.344 \\
=0.829 \\
=0.564 \\
=1.500\end{array}$ & $\begin{array}{l}\text { SIS (USA) } \\
\text { PИНЦ (Russia) } \\
\text { ESJI (KZ) } \\
\text { SJIF (Morocco) }\end{array}$ & $\begin{array}{l}=0.912 \\
=0.234 \\
=1.042 \\
=2.031\end{array}$ & $\begin{array}{l}\text { ICV (Poland) } \\
\text { PIF (India) } \\
\text { IBI (India) }\end{array}$ & $\begin{array}{l}=6.630 \\
=1.940 \\
=4.260\end{array}$ \\
\hline
\end{tabular}

жидкости, $\mathrm{m}^{2} / \mathrm{c}^{2}, \quad k_{\text {reg. }}=\max (k, 0), \quad$ где $\quad k-$ кинетическая энергия турбулентности потока жидкости, $\mathrm{M}^{2} / \mathrm{c}^{2}$.

Под относительной скоростью понимают скорость течения жидкости в пограничном слое

$$
v_{r e l .}=\left(u \cdot n_{x}+v \cdot n_{y}+w \cdot n_{z}\right)\left(u \cdot n_{x}+v \cdot n_{y}+w \cdot n_{z}\right),
$$

где первый множитель в уравнении промежуточная переменная для скорости течения жидкости на внутренней стенке модели круглой трубы $u_{n}<0$.

Составляющие среднего вязкостного напряжения на внутренней стенке круглой трубы $\tau_{\mu}\left(\mathrm{H} / \mathrm{MM}^{2}\right)$ от воздействия жидкости (когда сила трения жидкости будет равна силе сопротивления) можно представить системой уравнений (8)

$$
\left\{\begin{array}{l}
\tau_{\mu x}=\tau_{x x} \cdot n_{x}+\tau_{x y} \cdot n_{y}+\tau_{x z} \cdot n_{z} \\
\tau_{\mu y}=\tau_{y x} \cdot n_{x}+\tau_{y y} \cdot n_{y}+\tau_{y z} \cdot n_{z}, \\
\tau_{\mu z}=\tau_{z x} \cdot n_{x}+\tau_{z y} \cdot n_{y}+\tau_{z z} \cdot n_{z}
\end{array}\right.
$$

где $\tau_{\mu x}, \tau_{\mu y}, \tau_{\mu z}$ - составляющие $\tau_{\mu}, \mathrm{H} / \mathrm{Mм}^{2} ; \tau_{x x}, \tau_{x y}, \tau_{x z}$, $\tau_{y x}, \tau_{y y}, \tau_{y z}, \tau_{z x}, \tau_{z y}, \tau_{z z}$ - составляющие тензора вязкостного напряжения [9], Н/ м $^{2}$.

Скорость вязкой диссипации кинетической энергии турбулентности потока жидкости на внутренней стенке круглой трубы $\varepsilon_{w}\left(\mathrm{M}^{2} / \mathrm{c}^{3}\right)$ находится по формуле (9). относительно соседнего слоя. Относительная скорость течения жидкости на внутренней поверхности круглой трубы $v_{\text {rel }}$ (м/с) выражается формулой (7).

$$
\varepsilon_{w}=\frac{C_{\mu} \cdot k_{r e g}^{2} \cdot[\rho]}{\kappa_{v} \cdot \delta_{w}^{+} \cdot[\mu]}
$$

$\varepsilon_{w}$ увеличивается от установившегося потока жидкости до вихревого с максимумом на стенке трубы.

\section{Conclusion}

Расчетные значения параметров течения жидкости можно получить при построении в трехмерном пространстве (отверстие трубы) цветовых графиков. Предусмотрена генерация анимации для отображения изменения величин гидродинамических параметров во времени. Характер течения жидкости в пограничном слое круглой трубы будет зависеть от изменения величины плотности и динамической вязкости жидкости (например, в условиях увеличения температуры), а так же ряда постоянных коэффициентов.

\section{References:}

1. (2016) Navier-Stokes equations. Available: https://en.wikipedia.org/wiki/Navier-

Stokes equations (Accessed: 13.10.2016).

2. Chemezov D, Palev N (2016) Analytical models of the turbulent fluid flow in a circular pipe. ISJ Theoretical \& Applied Science, 09 (41): 77-84. Soi: http://s-o-i.org/1.1/TAS-0941-12

Doi: http://dx.doi.org/10.15863/TAS.2016.09.41.12

3. Sondak DL (1992) Wall functions for the k [epsilon] turbulence model in generalized nonorthogonal curvilinear coordinates. Retrospective Theses and Dissertations. Paper 9954. - 177 p.

4. (2016) Von Kármán constant. Available: https://en.wikipedia.org/wiki/Von_Kármán_con stant (Accessed: 13.10.2016).
5. (2015) Law of the wall. Available: https://en.wikipedia.org/wiki/Law_of the_wall (Accessed: 13.10.2016).

6. COMSOL Multiphysics User's Guide, 2010. $804 \mathrm{p}$.

7. (2016) Machine epsilon. Available: https://en.wikipedia.org/wiki/Machine_epsilon (Accessed: 13.10.2016).

8. (2016) K-epsilon turbulence model. Available: https://en.wikipedia.org/wiki/Kepsilon turbulence_model

(Accessed: 13.10.2016).

9. (2016) Viscous stress tensor. Available: https://en.wikipedia.org/wiki/Viscous_stress_te nsor (Accessed: 13.10.2016). 